Jesusa M. Santos, MD

Elias T. Reala, MD

Department of Otorhinolaryngology

Head and Neck Surgery

Jose R. Reyes Memorial Medical Center

Correspondence: Dr. Elias T. Reala

Department of Otorhinolaryngology

Head and Neck Surgery

Jose R. Reyes Memorial Medical Center

San Lazaro Compound, Rizal Avenue

Sta. Cruz, Manila 1003

Philippines

Phone: (632) 711-9491 local 320

E-mail: eliboy_entdoc@yahoo.com

The authors declare that this represents original material that is not being considered for publication or has not been published or accepted for publication elsewhere, in full or in part, in print or electronic media; that the manuscript has been read and approved by all the authors, that the requirements for authorship have been met by each author, and that each author believes that the manuscript represents honest work. Verbal consent was obtained from the patient for publication of this case report and accompanying images.

Disclosures: The authors signed disclosures that there are no financial or other (including personal) relationships, intellectual passion, political or religious beliefs, and institutional affiliations that might lead to a conflict of interest.

Presented at the Philippine Society of Otolaryngology Head and Neck Surgery Interesting Case Contest (2nd Place). May 12, 2018. Citystate Asturias Hotel, Puerto Princesa, Palawan.

\section{Tuberculosis of the Temporomandibular Region}

\begin{abstract}
Objective: To describe a unique case of extrapulmonary tuberculosis (TB) of the temporomandibular area focusing on its insidious and destructive course over a 2-year period with insights into the diagnostic and therapeutic pitfalls encountered throughout its clinical development.
\end{abstract}

\section{Methods:}

$\begin{array}{ll}\text { Design: } & \text { Case Report } \\ \text { Setting: } & \text { Tertiary Government Hospital } \\ \text { Patient: } & \text { One }\end{array}$

Results: A 33-year-old man initially presented with right pre-auricular swelling and trismus that were unresponsive to antibiotic therapy. On subsequent follow-ups, initial symptoms were accompanied by a non-healing right pre-auricular wound, right ear discharge, trismus and right facial paralysis (House-Brackmann III). Cranial and temporal bone computed tomography scans revealed osteolytic destruction of the right temporomandibular region extending to the auditory canal and of the right mastoid bone extending to the right mandibular condyle and parotid. Infected malignancy of the parotid, mandible and temporal bone were considered but definitive diagnosis from an incision biopsy revealed caseating granulomatous inflammation consistent with tuberculosis. He was started on anti-tuberculosis medications with significant resolution of pre-auricular swelling, non-healing pre-auricular wound, facial paralysis and ear discharge but minimal improvement in mouth opening.

Conclusion: Tuberculosis of temporomandibular region is rare and is associated with nonspecific manifestations. Delay in diagnosing and initiating appropriate treatment can lead to morbidity and serious complications involving destruction of the temporal bone, middle ear, mandible and parotid gland over its progression. A high index of suspicion by the physician and awareness of the patient's health seeking behaviors could have aided in the early diagnosis and treatment of this extrapulmonary TB.

Keywords: tuberculosis, temporomandibular region, pre-auricular swelling, trismus, antituberculosis therapy, facial paralysis, chronic mastoiditis, ear discharge 


\section{CASE REPORTS}

Tuberculosis (TB) remains a major public health problem worldwide and the impact of its magnitude can be felt by the continuing burden in the Philippines spanning centuries of high prevalence. Globally, there were an estimated 10.4 million new TB cases with 1.3 million TB deaths; estimates in the Philippines report an incidence rate of 554 per 100,000 and death rate of 21 per 100,000 population. ${ }^{1}$

It is common medical knowledge that TB most commonly affects the lungs. Extra-pulmonary TB comprises 15 20\% of the total TB infection burden. Of all cases of extrapulmonary TB, it is estimated that only $10 \%$ involve the head and neck region with predominance of cervical lymph node affectation (more than $90 \%$ of cases)., ${ }^{2,3}$ Temporomandibular TB is rare ${ }^{4}$ and we found no previous reports in HERDIN, the Philippine Journal of Internal Medicine and Philippine Journal of Otolaryngology Head and Neck Surgery.

This report aims to describe a case of extrapulmonary TB of the temporomandibular area, its clinical course over a 2-year period and provide insights into the diagnostic and therapeutic pitfalls encountered during its clinical development.

\section{CASE REPORT}

A 33-year-old man initially presented with 6-month history of initially non-painful right pre-auricular swelling and a 2-month history of gradual limitation of mouth opening. Physical examination showed a $2 \times 2 \mathrm{~cm}$, tender soft tissue swelling over the pre-auricular area, limitation of the mouth opening, palpable bilateral cervical lymph nodes and intact tympanic membranes. No cough nor afternoon fevers were reported. Initial clinical impression was acute bacterial parotitis versus infected first branchial cleft cyst prompting treatment with oral Coamoxiclav for 1 week, shifted to Clindamycin for another week with no resolution of the swelling. Imaging of the parotid area was requested but the patient was lost to follow-up.

After 16 months, the patient consulted with a general practitioner due to increasing limitation of mouth opening and extension of the pre-auricular swelling to the right temporal and infra-auricular areas. The patient also reported a 1-month history of right ear discharge and appearance of a non-healing wound over the right pre-auricular area. Plain Cranial Computed Tomography (CT) scan was interpreted as "soft tissue swelling and abscess formation in the scalp overlying the right temporal bone, chronic mastoiditis with no intracranial pathology and intact parotid glands."

The patient followed up at our institution with these results. Aside from the extended temporomandibular swelling, discharging ulcerations over the right pre-auricular area were noted on physical examination. (Figure $1 A, B)$ No symptoms referable to pulmonary pathology were elicited at this time except for intermittent undocumented fever. He denied any previous history of ear infection or discharge, diabetes mellitus, trauma to the affected areas and pulmonary tuberculosis.

Physical examination also showed deviation of mouth opening to the right with trismus documented as maximum inter-incisor distance of $26 \mathrm{~mm}$. (Figure 2) He also had ipsilateral peripheral facial nerve
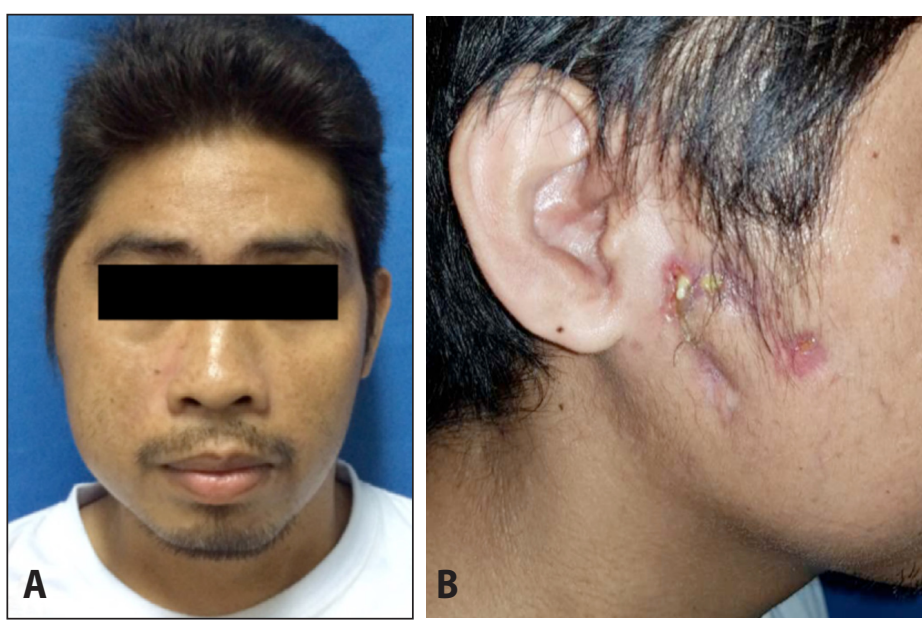

Figure 1A. Right pre-auricular swelling, and B. Non-healing right pre-auricular wound

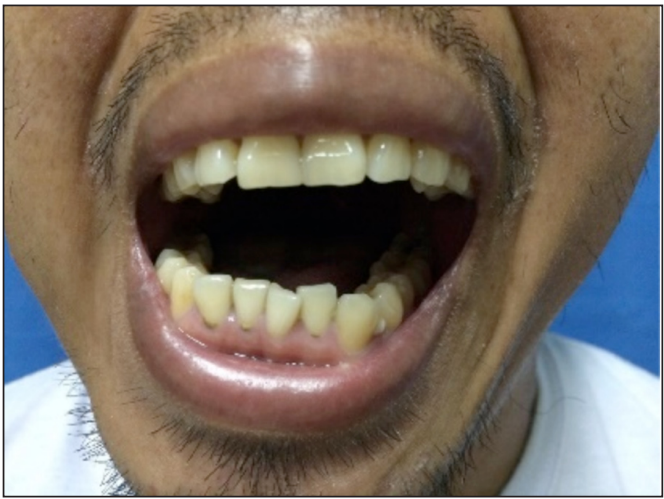

Figure 2. Note deviation of mouth opening to the right with limitation of mouth opening (maximum inter-incisor distance of $26 \mathrm{~mm}$ )

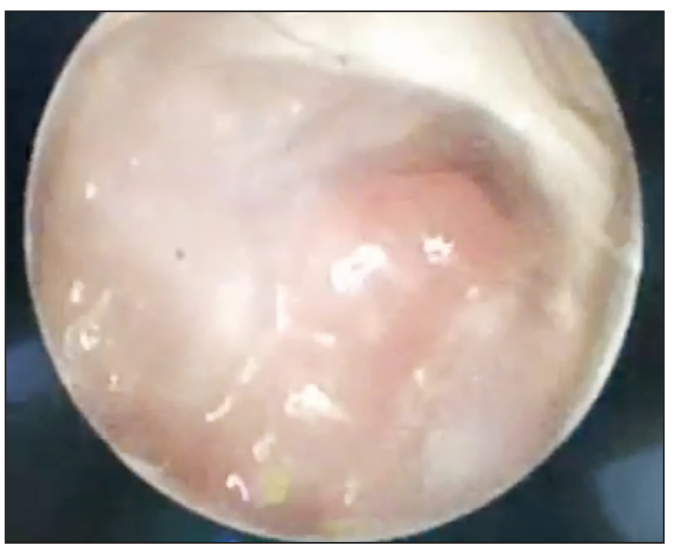

Figure 3. Otoscopic view showing right external auditory canal granulation tissue 
CASE REPORTS
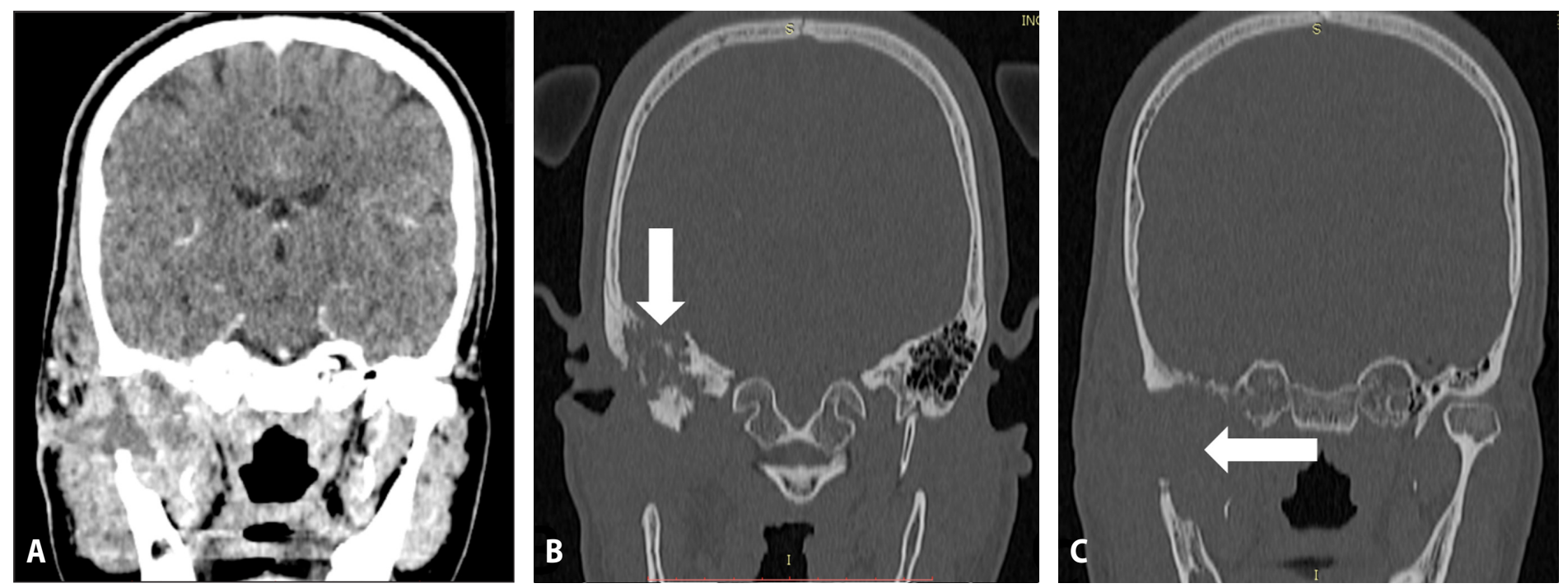

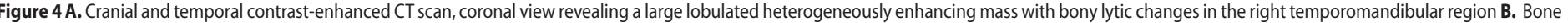
window showing osteolytic destruction of right temporal bone (arrow) and C. Osteolytic destruction of the right mandibular condyle, neck and ramus (arrow)

paralysis (House-Brackmann III). Otoscopy revealed granulation tissue with yellowish discharge occupying the right external auditory canal. (Figure 3)

An infected malignant neoplasm involving the temporal bone was highly considered when a repeat contrast-enhanced CT scan showed a large lobulated heterogeneously enhancing mass with bony lytic changes in the right temporomandibular region, with extension to the right buccal, masticator spaces, right parotid gland, mastoid air cells, middle and outer ear cavities. Osteolytic destruction of the right mandibular condyle, coronoid process, neck and ramus, the tympanic, horizontal and zygomatic part of the right temporal bone, right mastoid bone, and erosion of the tegmen mastoideum and tegmen tympani were also evident. (Figure $4 \mathrm{~A}-\mathrm{C}$ )

Punch biopsy of the external auditory canal mass, fine needle aspiration biopsy (FNAB) of the pre-auricular area and temporal area swellings revealed granulation/necrotic tissue and the absence of malignant cells. Subsequently, a deeper incision biopsy over the non-healing wound in the pre-auricular area was read as caseating granulomatous inflammation consistent with tuberculosis. (Figure 5)

He was referred to our TB Direct Observed Treatment, ShortCourse (DOTS) clinic and started on anti-tuberculosis medications. One month into taking anti-TB medications, the patient reported complete resolution of the right ear discharge, skin ulcerations and swelling on the temporal and pre-auricular area. However, only partial resolution of the facial muscle weakness, and minimal resolution of trismus / difficulty of opening of the mouth was reported. Unfortunately, after these improvements, our patient subsequently went home to Samar province and was lost to follow-up.

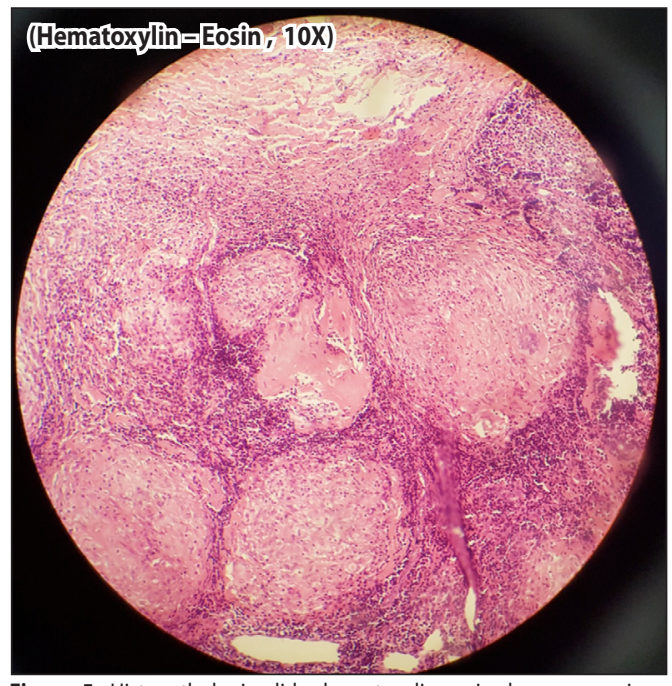

Figure 5. Histopathologic slide, hematoxylin-eosin, low-power view (10X) showing caseating granulomatous inflammation consistent with tuberculosis

\section{DISCUSSION}

We described a patient with extrapulmonary TB of the temporomandibular area, its insidious natural course over 2 years, and aim to provide insights into the diagnostic and therapeutic pitfalls encountered throughout its clinical development.

It is generally thought that most extrapulmonary TB results from hematogenous dissemination of the Mycobacterium..$^{5}$ Other modes of dissemination are direct transfer, regional extension of soft tissue lesions, lymphatic routes, or a combination of these modes. The patient's TB probably started in a pre-auricular lymph node outside the parotid. How the mycobacteria arrived in the area cannot be singled 


\section{CASE REPORTS}

Philippine Journal Of Otolaryngology-Head And Neck Surgery

Vol. 33 No. 2 July - DeCEMBER 2018

PJOHE

out although the presence of palpable multiple bilateral non- tender cervical nodes does provide clues to this. The late administration of effective anti-TB medications allowed us to describe the path of spread and destruction the disease took in this patient. From the subcutaneous, superficial parotid pre-auricular node, the disease manifested as a slow growing 6-month swelling which spread to involve the structures surrounding the temporomandibular joint, hence the 2-month history of trismus. From there, and over the course of 15 months, the lesion spread to involve the mastoid air cells and middle ear resulting in a discharging ear. The skin over the initial site followed next and manifested as scrofuloderma. Just a few weeks after the initial CT scan reported the parotid being intact, the disease breached the parotid fascia and penetrated the parotid gland thereby affecting the upper branches of the facial nerve. The repeat CT scan also revealed widespread involvement of adjacent bony structures, obliterating parts of the mandibular ramus and condyle, infiltrating the mastoid on the verge of breaching the intracranial space. While anti-TB therapy reversed many manifestations of the disease, the long-term or permanent impact of TB on the patient's mastication and upper facial nerve functions remains to be seen.

Tuberculosis can affect any organ system in the body. It has been called "the great mimicker" because of its ability to simulate a number of other disease entities depending on the organ involved. ${ }^{6}$ Diagnosing extrapulmonary tuberculosis is often difficult since many patients present with nonspecific symptoms, negative purified protein derivative skin test and negative culture of specimens. ${ }^{5}$ The nonspecific signs and symptoms presented by our patient were trismus, ear mass, ear discharge, and facial nerve paralysis which are similar to those of inflammation or infection, benign neoplasm and malignancy of the involved structures, hence, the diagnosis of TB was initially missed.

A high index of suspicion and familiarity with the local incidence is needed in order to facilitate the diagnosis of extrapulmonary TB. The clues were there and can be deduced relatively easily on hindsight. The multiple cervical lymphadenopathies, intermittent fever and appearance of scrofuloderma. The indolent nature of this disease led to a burst of hurried deterioration.

It is important to consider tuberculosis of temporomandibular region in a patient with unusual presentation of pre-auricular swelling and trismus despite the absence of classic symptoms associated with pulmonary $\mathrm{TB}^{7}$ since we are living in a country with high TB prevalence. The diagnostic tests that should have been employed earlier consist of culture of Mycobacterium from bone tissue, AFB smear, fine-needle aspiration cytology and tissue biopsy. ${ }^{5}$ However, almost $40-50 \%$ of patients will have no evidence of tuberculosis elsewhere ${ }^{6}$ and the repeated biopsies of our patient were inconclusive. Histopathologic examination is needed to make a definitive diagnosis. The CT scan was also helpful in showing the extent of disease but imaging alone is insufficient in reaching a conclusive diagnosis.

The patient was lost to follow up early in the clinical management of his complaints and returned only after 16 months had passed with a plethora of rapidly deteriorating new complaints. Indeed, socioeconomic issues bear greatly on a patient's consults and follow-up. In fact, following the clinical improvements reported in this case, he went back to his hometown and was again lost to follow up.

Needless to say, experience has yet again taught us that prompt recognition and treatment is key to reverse the symptoms and arrest the progression of TB. Any delays in treatment may allow progression to significant TMJ destruction, total facial nerve paralysis, intracranial infection and irreversible hearing loss. Anti-tuberculosis therapy should be started and completed for at least 6 months, identical to the treatment of pulmonary TB.7 Surgical interventions in the absence of appropriate medical therapy may result in complications such as fistula, non-healing suture lines and failure of surgery. Since our patient presented with large abscess formation and osteolytic changes in the temporomandibular region, possible surgical excision, decortication and joint reconstruction ${ }^{8}$ may have been considered if anti-tuberculosis therapy failed. ${ }^{8}$ Together with all these, socio-economic determinants of health must be addressed on a systematic level, or we risk the relapse of our patient, and occurrence in others.

In summary, we described a rare case of extrapulmonary TB of the temporomandibular area manifested by trismus, right pre-auricular swelling, scrofuloderma, right ear discharge and right facial paralysis that developed over a span of 2 years. These are common findings in otolaryngology with a multitude of differential diagnoses covering the parotid, mandible and temporal bone. A thorough clinical history, physical examination and high index of suspicion are needed for the diagnosis and treatment of this elusive but very common disease in an uncommon location.

\section{REFERENCES}

1. World Health Organization. Global Tuberculosis Report 2017: Executive Summary. [cited 2017 Nov 13]. Available from: http://www.who.int/tb/publications/global_report/Exec Summary_13Nov2017.pdf.

2. Nalini B, Vinayak S. Tuberculosis in ear, nose, and throat practice: its presentation and diagnosis Am J Otolaryngol. 2006 Jan-Feb; 27(1): 39-45. DOI: 10.1016/j.amjoto.2005.07.005; PMID: 16360822.

3. Prasad KC, SreedharanS, ChakravarthyY,PrasadSC.Tuberculosis in the head and neck:experience in India. J Laryngol Otol. 2007 Oct; 121(10): 979-85. DOI: 10.1017/S0022215107006913; PMID: 17367564.

4. Kreiner M. Tuberculosis of the temporomandibular joint: low prevalence or missed diagnosis? Cranio. 2006 Oct; 24(4):234. PMID: 17086851.

5. Sharma SK, Mohan A. Extrapulmonary tuberculosis. Indian J Med Res. 2004 Oct: 316-53. PMID: 15520485.

6. Harisinghani MG, McLoud TC, Shepard JA, Ko JP, Shroff MM, Mueller PR. Tuberculosis from head to toe. Radiographics. 2000 Mar-Apr; 20(2): 449-470; quiz 528-529, 532. DOI: 10.1148/ radiographics.20.2. g00mc12449; PMID: 10715343

7. World Health Organization. Global tuberculosis control: WHO report 2011. Geneva: World Health Organization, 2011:1-258. [cited 2017 Nov 13]. Available from: http://www.who.int/iris/ handle/10665/44728.

8. Patel M, Scott N, Newlands C. Case of tuberculosis of the temporomandibular joint. Br J Oral Maxillofac Surg. 2012 Jan; 50(1): e1-3. DOI: 10.1016/j.bjoms.2011.05.012; PMID: 21676508 